\title{
Case-Control Study on TNFRSF6B Gene Polymorphism and Susceptibility to Gastric Cancer in a Chinese Han Population
}

This article was published in the following Dove Press journal: Pharmacogenomics and Personalized Medicine

Xuyu Gu (D)

Zhenwei Mao ${ }^{2}$

Huiwen $\operatorname{Pan}^{2}$

Chen Zou ${ }^{2}$

Guowen Ding ${ }^{2}$

Yu Fan ${ }^{2}$

'School of Medicine, Southeast University, Nanjing, Jiangsu 210009 . People's Republic of China; ${ }^{2}$ Cancer Institute, Affiliated People's Hospital of Jiangsu University, Zhenjiang, Jiangsu 212002, People's Republic of China
Correspondence: Yu Fan; Guowen Ding Cancer Institute, Affiliated People's Hospital of Jiangsu University, Zhenjiang, Jiangsu 2I2002, People's Republic of China Email yufl I I223@sina.com; dingguowen2013@I63.com
Purpose: To explore the relationship between rs2297440 and rs2297441 polymorphisms of TNFRSF6B gene and susceptibility to gastric cancer.

Methods: A hospital-based case-control study was conducted. A total of 577 gastric cancer cases and 678 normal controls were recruited. Their genotypes were determined using the SnapShot method.

Results: The smoking rate in the case group (34.49\%) was higher than that in the control group (27.29\%). For TNFRSF6B rs2297440, among people $<62$ years old, the risk of gastric cancer in TC people was 1.84 times that in TT people. Among the non-drinking people, the risk of gastric cancer in the CC type was 0.66 times that in the TT+TC type. Among the drinking population, the risk of gastric cancer in the TC type was 1.67 times that in the TT type, and the risk in the TC+CC type was 1.70 times that in the TT type. As for TNFRSF6B rs2297441, in males and non-drinkers, the risk of gastric cancer in the AG type was less than that in the GG type. No matter how old the patient is, the risk of gastric cancer in the AA type was less than that in the $\mathrm{AG}+\mathrm{GG}$ type.

Conclusion: A correlation exists between smoking and gastric cancer. For TNFRSF6B rs2297440, the TC genotype may be a risk factor for gastric cancer in people $<62$ years old. In the non-drinking population, the homozygous mutant of $\mathrm{CC}$ may be a protective factor for gastric cancer. In the drinking population, TC type may be a risk factor, whereas the TC+CC type dominated by $\mathrm{C}$ may be a protective factor. For TNFRSF6B rs2297441, the AG genotype may be a risk factor for gastric cancer in males and non-drinkers. The AA homozygous mutant may be a protective factor for gastric cancer.

Keywords: gastric cancer, TNFRSF6B gene, single nucleotide polymorphism, rs2297440, rs2297441

\section{Introduction}

Gastric cancer (GC) is the fifth most common cancer and the third most common cause of cancer death globally. ${ }^{1}$ In the East, GC is often diagnosed in its early stages in countries, such as Japan and Korea, but more than $80 \%$ of patients with GC in China are already in advanced stages at the time of diagnosis. ${ }^{2}$ The traditional treatments of surgery and chemoradiotherapy in the late stage of the disease are not as effective as those in early stage of the disease because of the malignant invasion and metastasis that occur in later stages, and the 5-year survival rate is $<20 \%$. $^{3}$ The reasons can be considered as follows. On the one hand, it is the result of insidious onset. On the other hand, it could be the lack of effective diagnostic 
molecular markers in the early period nowadays. ${ }^{4}$ Accordingly, molecules important to the growth of GC should be identified to improve the early diagnosis and treatment of the disease.

Exploratory development of the pathogenesis of GC improves with the development of third-generation genetic marker technology. Single nucleotide polymorphism (SNP) analysis is commonly used in the research of complex diseases and genetic identification of the population. ${ }^{5}$ The research of tumor-associated gene polymorphisms may bring new predictive markers for cancer treatment.

Tumor necrosis factor receptor superfamily member 6B (TNFRSF6B) is also known as decoy receptor 3 (DcR3), M68, or tumor necrosis receptor factor 6(TR6). ${ }^{6}$ TNFRSF $6 B$ is a member of the tumor necrosis factor receptor family, a type of receptor protein molecule that can specifically bind to certain protein ligands. ${ }^{7}$ TNFRSF $6 B$ is significantly increased in most malignant tumors, such as colon cancer, ${ }^{8}$ liver cancer, ${ }^{9}$ esophageal cancer, ${ }^{10}$ lung cancer, ${ }^{11}$ glioma, ${ }^{12}$ lymphoma, ${ }^{13}$ pancreatic cancer, ${ }^{14}$ renal cell cancer, ${ }^{15}$ and breast cancer. ${ }^{16}$ In these malignant tumors, amplification of the TNFRSF6B gene and high expression of mRNA or protein can be detected. The status of TNFRSF6B is closely related to the invasion and metastasis of malignant tumors. ${ }^{17}$ Therefore, TNFRSF $6 B$ expression is a potential tumor marker for the early detection or prognosis of malignant tumors. The regulatory role of these SNPs must be investigated to understand the role of TNFRSF $6 B$ in tumor cells. The promoter of the TNFRSF6B gene contains two SNPs, rs2297441 (-539 G/A) and rs2257440 (147 C/T), which are common variants of Han Chinese. ${ }^{10}$ Xiong et al ${ }^{18}$ found that the SNP rs2257440, present in exon 1 of $D c R 3$, is significantly associated with the susceptibility of esophageal cancer. Dai et $\mathrm{al}^{19}$ further found that rs2257440 is a functional SNP and that the T allele of rs2257440 can increase DcR3 expression because it promotes binding of the gene with the specific transcription factor MTF-1. Fu et $\mathrm{al}^{20}$ found that DcR3 gene polymorphisms are associated with sporadic breast infiltrating ductal carcinoma risk in northeast Chinese females.

However, relevant research on the polymorphism between TNFRSF6B and GC is lacking. Thus, we performed a case-control study to compare the genotypes and alleles of TNFRSF6B genes rs2297440 and rs2297441 in patients with GC and in healthy controls to analyze the relationship between TNFRSF6B polymorphism and GC susceptibility. We combined the patients' clinical parameters, such as gender, age, smoking history, and drinking history, to analyze the correlation between them comprehensively. This study provides a theoretical basis for the early screening and early treatment of GC in the future.

\section{Patients and Methods}

The case group was composed of 577 GC patients (394 males and 183 females) treated at the People's Hospital of Jiangsu University from May 2013 to June 2017. The patients in this group had an average age of 61.34 \pm 11.097 years. All patients were Chinese Han population and were diagnosed as primary gastric cancer by clinical and pathological confirmation. Patients with secondary or recurrent tumors and other malignant tumors were excluded. None of the patients received radiotherapy or chemotherapy. The control group was composed of 678 healthy individuals (456 males and 222 females) from the Physical Examination Center of the People's Hospital of Jiangsu University during the same period. The patients in this group had an average age of $62.31 \pm 7.549$ years. All healthy controls had no history of tumor genetic disease. The control and case groups matched the gender and age of the patients. The two groups are not related by blood. Exactly $2 \mathrm{~mL}$ of anticoagulated whole blood was extracted from all participants and centrifuged to extract plasma. The remaining whole blood was used to extract genomic DNA. Purity and concentration were detected using a protein nucleic acid detector.

The PCR products were purified with ExoI and FastAP and then subjected to extension reaction. Combined with the NCBI database, the upstream and downstream primers and snapshot extension primers at the rs2297440 and rs2297441 positions of the TNFRSF6B gene were designed, and the primers were synthesized by Free biotech (Shanghai) Co., Ltd. TNFRSF6B rs2297440 forward: 5'-AAACACCCACCTACCCCTGG -3',TNFRSF6B rs2297440 reverse: 5'-AGAACTGCGTGTAGTGGCG-3', TNFRSF6B rs2297441 forward: 5'-CTCACCGGG AAGGAGGAGA -3', TNFRSF6B rs2297441 reverse:5 'CCAACTCCCTGTCCCTCAAC - 3 '. Human $\beta$-actin was used as an endogenous control. For each sample, the difference in threshold cycle was calculated using the $2^{-\triangle \Delta C T}$ method. ABI3730XL sequencer was used for sequencing and genotyping. The Snapshot method was used for genetic polymorphism detection. After the test was completed, $5 \%$ samples were randomly selected for re-testing to ensure the accuracy of the test results. The 
research was approved by the Ethical Review Committee of Jiangsu University, and the project fund was confirmed.

\section{Statistical Analysis}

The distributions of demographic characteristics, selected variables, and genotypes of the TNFRSF $6 B$ gene variant differences between the case and control groups were evaluated using the $\chi^{2}$ test. Logistic regression analyses were used to estimate the associations between the SNPs and the risk of GC for crude and adjusted ORs when adjusting for age, sex, smoking, and drinking status. Bonferroni correction was also applied because of the number of comparisons. The Hardy-Weinberg equilibrium (HWE) was tested using the goodness-of-fit $\chi^{2}$ test to compare the observed genotype frequencies to the expected ones among the controls. All statistical analyses were performed on SPSS 20.0 (SPSS Inc., Chicago, Illinois, USA).

\section{Results}

Table 1 lists the essential information on the TNFRSF6B gene rs2297440 and rs2297441 polymorphisms. The genotype frequency distribution of the selected samples conforms to the HWE and is representative.

Table 2 shows the features of the study subjects, including demographic and environmental factors. Results of $\chi^{2}$ test showed that the case and control groups were well matched in terms of age and sex. As presented in Table 2, a huge disparity in smoking status $(P=0.006)$ was discovered between the case and control groups. The rate of alcohol drinking was lower in the case group than in the control group, but the difference was not significant ( $21.49 \%$ vs $23.30 \%, P=0.443$ ).

As shown in Table 3, when wild type genotype TT served as the reference, the recurrence rate of TC heterozygous variations at the rs 2269700 locus was higher in the case group than in the control group $(48.60 \%>42.86 \%)$, but no evident disparity was observed between these two groups $(P=0.097)$. After logistic regression adjustment analysis, no disparity in data was observed, but the result approached the difference more than before $(P=0.067)$. The rate of recurrence distribution of $\mathrm{CC}$ homozygous mutants in the case group was lower than that in the control group $(9.97 \%<12.48 \%)$. However, the difference in data was not significant. After logistic regression adjustment analysis, no disparity in data was observed $(P=0.622)$. In the dominant model, the rate of recurrence distribution of $\mathrm{TC}+\mathrm{CC}$ variations in the case group was higher than that in the control group $(58.57 \%>55.37 \%)$.

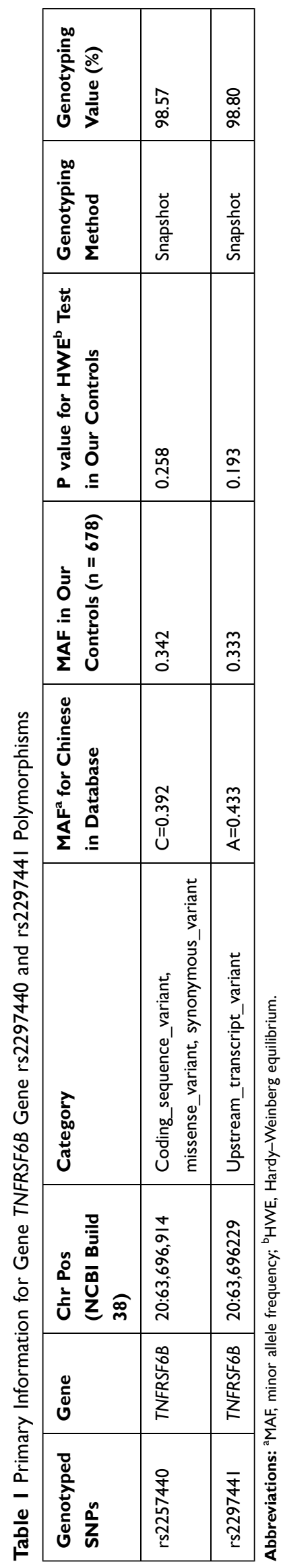


Table 2 Distribution of Selected Demographic Variables and Risk Factors in GC Cases and Controls

\begin{tabular}{|c|l|l|l|}
\hline \multirow{2}{*}{ Factors } & $\begin{array}{l}\text { Overall Cases } \\
(\mathbf{n = 5 7 7 )}\end{array}$ & $\begin{array}{l}\text { Overall Controls } \\
(\mathbf{n = 6 7 8 )}\end{array}$ & $\boldsymbol{P}$ \\
\cline { 2 - 3 } & $\mathbf{n}(\%)$ & $\mathbf{n}(\%)$ & 0.065 \\
\hline Age (years) & $61.34 \pm \mathrm{II} .097$ & $62.3 \mathrm{I} \pm 7.549$ & \\
\hline $\begin{array}{c}\text { Age (years) } \\
<62 \\
\geq 62\end{array}$ & $\begin{array}{l}268(46.45) \\
309(53.55)\end{array}$ & $\begin{array}{l}324(47.79) \\
354(52.21)\end{array}$ & 0.635 \\
\hline $\begin{array}{c}\text { Sex } \\
\text { Male } \\
\text { Female }\end{array}$ & $\begin{array}{l}394(68.28) \\
183(31.72)\end{array}$ & $\begin{array}{l}456(67.26) \\
222(32.74)\end{array}$ & 0.698 \\
\hline $\begin{array}{c}\text { Smoking status } \\
\text { Never } \\
\text { Ever }\end{array}$ & $\begin{array}{l}378(65.5 \mathrm{I}) \\
199(34.49)\end{array}$ & $\begin{array}{l}493(72.71) \\
185(27.29)\end{array}$ & 0.006 \\
\hline $\begin{array}{c}\text { Alcohol use } \\
\text { Never } \\
\text { Ever }\end{array}$ & $\begin{array}{l}453(78.5 \mathrm{I}) \\
124(2 \mathrm{I} .49)\end{array}$ & $\begin{array}{l}520(76.70) \\
158(23.30)\end{array}$ & 0.443 \\
\hline
\end{tabular}

The difference in data was not significant $(P=0.253)$. After logistic regression adjustment analysis, the result approach the difference more than before $(P=0.202)$. In the distribution of recessive model dominated by the $\mathrm{T}$ allele, the rate of recurrence distribution in the case group was lower than that in the control group $(90.03 \%<87.52 \%)$. No disparity in statistical data was observed $(P=0.164)$. After logistic regression adjustment analysis, no difference in statistical data was observed $(P=0.186)$.

As demonstrated in Table 4, the analyses proved that the genotype frequencies of TNFRSF6B genes rs2297441 and rs2257440 did not significantly differ between the two groups $(P>0.05)$. These four polymorphic sites show no relevance with the danger of $\mathrm{GC}$ as depicted by the logistic regression analysis.
As shown in Table 5, haplotype analysis revealed no obvious difference in the frequencies of the rs 2257440 and rs2297441 alleles between the groups $(P=0.852, P=0.619)$.

Furthermore, stratification analyses were performed to evaluate the effects of the TNFRSF $6 B$ gene rs 2257440 on the risk of GC in consideration of age, gender, smoking, or alcohol drinking (Table 6 ). In the younger cohort ( $<62$ years), the recurrence frequency rate of the TC genotype was various in data between these groups $(P=0.028)$, and the danger of $\mathrm{GC}$ in the TC genotype was 1.48 times that in the TT genotype. The TC genotype could be a danger factor. When treating the nondrinking group, the rate of recurrence distribution of CC homozygous mutations was also various in the recessive model ( $P=0.043$ ), and the danger of $\mathrm{GC}$ in the $\mathrm{CC}$ type was 0.66 times that in the TT+TC type. The CC homozygous variation type may be a protective factor for GC. Referring to drinking group, the rate of recurrence distribution of TC was various in statistical data in these groups $(P=0.049)$, and the danger of GC in the TC type was 1.67 times that in the TT type. The TC type could be a dangerous element. In the model dominated by $\mathrm{C}$, the rate of recurrence distribution of $\mathrm{TC}+\mathrm{CC}$ was various in the $\mathrm{TC}+\mathrm{CC}$ and TT types $(P=0.033)$, and the danger of $\mathrm{GC}$ in the $\mathrm{TC}+\mathrm{CC}$ type was 1.70 times in the TT type, and the $\mathrm{C}$-dominated $\mathrm{TC}+\mathrm{CC}$ type may be a protective factor.

Stratification analyses were carried out to assess the effects of TNFRSF $6 B$ rs 2297441 on GC risk in consideration of gender, smoking, age, and alcohol drinking (Table 7). In the male group, the rate distribution of $A G$ was various between these groups $(P=0.011)$, and the danger of $\mathrm{GC}$ in the AG type was 1.45 times that in the GG type. The AG type could be a dangerous element. In the younger population of $<62$ years old, the rate distribution of AA homozygous alteration was also various in statistical data in the recessive model $(P=0.031)$, and the danger of GC in the AA type was 0.54 times that in the

Table 3 TNFRSF6B Gene rs2257440 Polymorphism in GC Cases and Controls and Logistic Regression Analysis

\begin{tabular}{|c|c|c|c|c|c|c|c|c|}
\hline \multirow[t]{2}{*}{ Genotype } & \multicolumn{2}{|c|}{$\begin{array}{l}\text { GC Cases } \\
(n=577)\end{array}$} & \multicolumn{2}{|c|}{$\begin{array}{l}\text { Controls } \\
(n=678)\end{array}$} & \multirow[t]{2}{*}{$\begin{array}{l}\text { Crude OR } \\
(95 \% \mathrm{Cl})\end{array}$} & \multirow[t]{2}{*}{$P$} & \multirow[t]{2}{*}{$\begin{array}{l}\text { Adjusted OR } \\
(95 \% \mathrm{Cl})\end{array}$} & \multirow[t]{2}{*}{$P$} \\
\hline & $\mathbf{n}$ & $\%$ & $\mathbf{n}$ & $\%$ & & & & \\
\hline \multicolumn{9}{|l|}{ rs2257440 } \\
\hline TT & 237 & 41.43 & 297 & 44.66 & 1.00 & & 1.00 & \\
\hline TC & 278 & 48.60 & 285 & 42.86 & $1.22(0.96-1.95)$ & 0.097 & $1.25(0.98-1.59)$ & 0.067 \\
\hline CC & 57 & 9.97 & 83 & 12.48 & $0.86(0.59-1.26)$ & 0.436 & $0.96(0.79-1.16)$ & 0.622 \\
\hline $\mathrm{TC}+\mathrm{CC}$ & 335 & 58.57 & 368 & 55.34 & $1.14(0.91-1.43)$ & 0.253 & $1.07(0.96-1.20)$ & 0.202 \\
\hline CC & 57 & 9.97 & 83 & 12.48 & $0.78(0.54-I . I I)$ & 0.164 & $1.08(0.96-1.21)$ & 0.186 \\
\hline $\mathrm{TT}+\mathrm{TC}$ & 515 & 90.03 & 582 & 87.52 & 1.00 & & 1.00 & \\
\hline
\end{tabular}


Table 4 TNFRSF6B Gene rs229744I Polymorphism in GC Cases and Controls and Logistic Regression Analysis

\begin{tabular}{|c|c|c|c|c|c|c|c|c|}
\hline \multirow[t]{2}{*}{ Genotype } & \multicolumn{2}{|c|}{$\begin{array}{l}\text { GC Cases } \\
(n=577)\end{array}$} & \multicolumn{2}{|c|}{$\begin{array}{l}\text { Controls } \\
(n=678)\end{array}$} & \multirow[t]{2}{*}{$\begin{array}{l}\text { Crude OR } \\
(95 \% \mathrm{Cl})\end{array}$} & \multirow[t]{2}{*}{$P$} & \multirow[t]{2}{*}{$\begin{array}{l}\text { Adjusted OR } \\
(95 \% \mathrm{Cl})\end{array}$} & \multirow[t]{2}{*}{$P$} \\
\hline & $\mathbf{n}$ & $\%$ & $\mathbf{n}$ & $\%$ & & & & \\
\hline \multicolumn{9}{|l|}{ Rs229744 I } \\
\hline GG & 240 & 42.33 & 315 & 46.81 & 1.00 & & 1.00 & \\
\hline AG & 276 & 48.68 & 280 & 41.60 & $1.29(1.02-1.64)$ & 0.033 & $1.32(1.04-1.67)$ & 0.025 \\
\hline AA & 51 & 8.99 & 78 & 11.59 & $0.86(0.58-1.27)$ & 0.443 & $0.95(0.78-1.16)$ & 0.592 \\
\hline$A G+A A$ & 327 & 57.67 & 358 & 53.19 & $0.20(0.96-1.50)$ & 0.114 & $1.10(0.99-1.24)$ & 0.091 \\
\hline AA & 51 & 8.99 & 78 & 11.59 & $0.75(0.52-1.09)$ & 0.136 & $0.87(0.72-1.05)$ & 0.142 \\
\hline $\mathrm{GG}+\mathrm{AG}$ & 516 & 91.01 & 595 & 88.41 & 1.00 & & 1.00 & \\
\hline
\end{tabular}

Table 5 Analysis of rs2257440 rs229744I Alleles Between Cases and Controls

\begin{tabular}{|l|l|l|l|l|l|}
\hline locus & Variable & Case & Control & $P$ & OR (95\% CI) \\
\hline rs2257440 & T allele & $752(65.73)$ & $879(66.09)$ & & \\
\cline { 2 - 6 } & C allele & $392(34.27)$ & $451(33.91)$ & 0.852 & $1.02(0.86-1.20)$ \\
\hline rs229744I & G allele & $756(66.67)$ & $910(67.61)$ & & \\
\cline { 2 - 6 } & A allele & $378(33.33)$ & $436(32.39)$ & 0.619 & $1.04(0.88-1.24)$ \\
\hline
\end{tabular}

AG+GG type. The AA homozygous mutant could be a protective element for GC. Similarly, the result in the older group ( $>62$ years) was the same as that in the younger group. In the non-drinking population, the frequency distribution of $\mathrm{AG}$ was various in statistical data in the two groups $(P=0.030)$, and the danger of $\mathrm{GC}$ in the $\mathrm{AG}$ type was 1.74 times that in the GG type. The AG type could be a dangerous element. In the dominant model, the $\mathrm{AG}+\mathrm{AA}$ rate distribution was various in the two groups $(P=0.009)$. The AG+AA type may be a risk factor.

\section{Discussion}

GC is a disease caused by multiple factors, including diet, gene, environment, immunity, infections, and inflammation. Such factors could result in certain signaling pathways out of balance, which closely refers to the progression of disease and the depression elements of GC. Lately, TNFRSF6B is a member of the tumor necrosis element receptor superfamily. TNFRSF6B is a soluble receptor that can bind and inactivate the apoptosis-inducing ligand FasL, LIGHT, and TL1A. ${ }^{21}$ Therefore, TNFRSF6B has the potential to prevent tumor cell apoptosis. By suppressing the function of macrophages and T-cells, ${ }^{22}$ TNFRSF6B can hinder LIGHT-triggered T-cell costimulation through HVEM and attune the interplay of immune cell. These features of TNFRSF6B play a role in helping tumor cells obtain a survival advantage and avoid immune monitoring. Other studies pointed out that TNFRSF6B is excessively expressed in many types of malignancies. However, studies on the association of the TNFRSF6B gene with GC are few. Edfeldt et $\mathrm{al}^{23}$ found that TNFRSF $6 B$ is related to poor survival and can be considered as a marker for liver metastases. $\mathrm{Hu}$ et $\mathrm{al}^{24}$ showed that the expression of $D c R 3$ is higher in the tumor tissues of GC than in paracarcinoma tissues and that TNFRSF6B is important in assessing the stage of GC.

Currently, studies on TNFRSF6B polymorphisms are rare. SNPs rs2297441 (-539 G/A) and rs2257440 (147 C/T) are common mutations in Chinese Han when treating the promotor TNFRSF6B gene. These two gene polymorphisms may alter the promoter activity of TNFRSF6B and may serve certain functions that affect an individual's susceptibility to cancer. A previous study $^{18}$ showed that the rs2257440 polymorphism of the TNFRSF6B gene is related to the danger of esophageal cancer and is strongly correlated with clinical TNM staging. Furthermore, the risk of the $147 \mathrm{CC}$ genotype was more pronounced among smokers, suggesting that smoking is an independent risk for esophageal cancer, which is consistent with our experimental results.

When dealing with this research, 577 patients with GC and 678 healthy volunteers were selected to explore the 


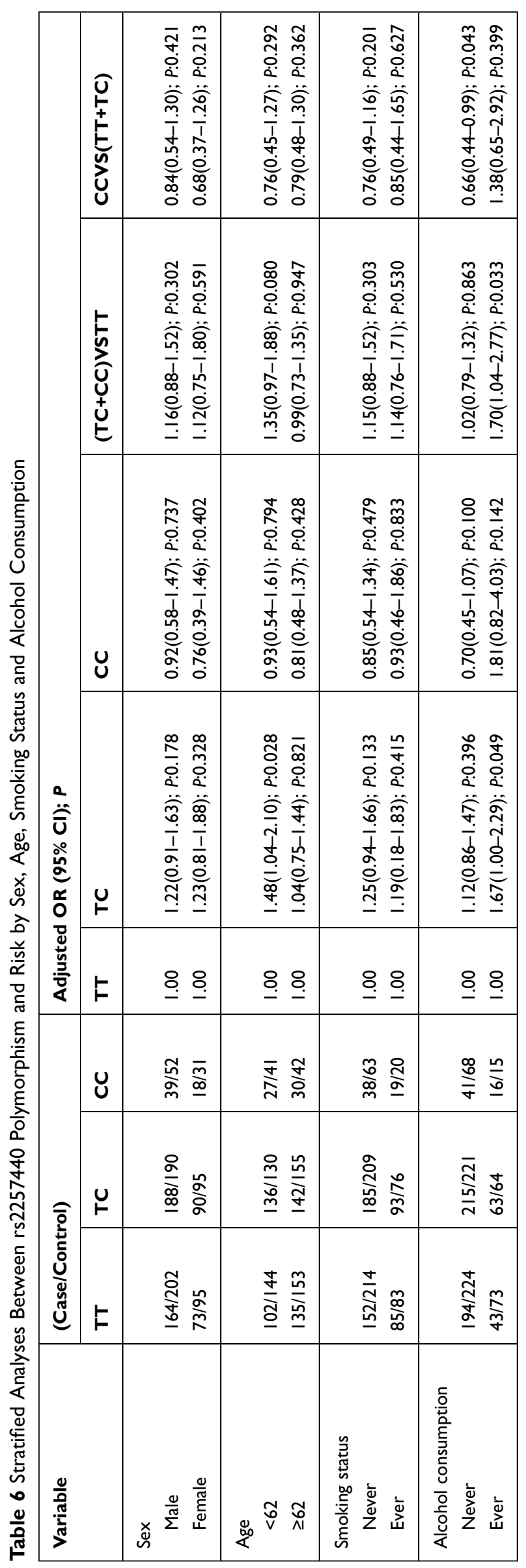

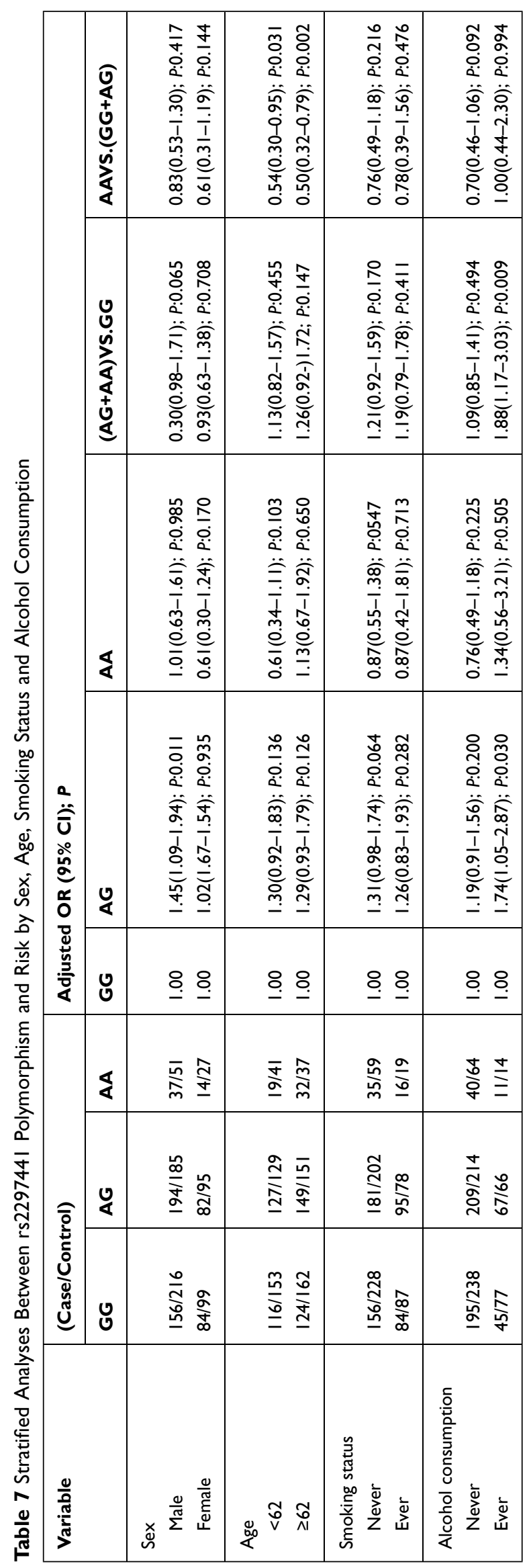


correlation between TNFRSF6B gene rs2297441 (-539 G/A) and rs2257440 (147 C/T) loci and GC susceptibility in Chinese Han's population. The frequency of smoking in the case group was $34.49 \%$, which was higher than that in the control group (27.29\%), and the distinction in rate distribution in the two groups was significant, indicating that smoking is related to the detection and aggravation of GC. Smoking can be treated as an individual factor when treating GC. However, no statistically visible variation in the distribution of drinking factors was found between the two groups.

The TNFRSF6B gene rs2257440 polymorphism was shown according to the stratified results. Regardless of age and drinking status, the rate of recurrence distribution of TC type was various in statistical data in these groups. The danger of GC in the TC type significantly exceeded that in the TT type, indicating that the TC type is a danger factor. Considering non-drinking population, the rate distribution of CC homozygous variations varied in the recessive model. The danger of $\mathrm{GC}$ in the $\mathrm{CC}$ type was 0.66 times that in the $\mathrm{TT}+\mathrm{TC}$ type. The $\mathrm{CC}$ homozygous mutant may be a protective factor of GC. Among the drinking population, the rate distribution of the $\mathrm{TC}+\mathrm{CC}$ and $\mathrm{TT}$ types differed between the two groups in the leading model dominated by C. The danger of $\mathrm{GC}$ in the $\mathrm{TC}+\mathrm{CC}$ type was 1.70 times that in the TT type. Thus, the $\mathrm{C}$-dominant $\mathrm{TC}+\mathrm{CC}$ type could be a protective element. The rs 2257440 polymorphism of the TNFRSF6B gene is associated with the danger of GC, and the $\mathrm{C}$ mutation may be a protective factor for GC.

The rs2297441 polymorphism of the TNFRSF6B gene in the rate distribution and the logistic regression analysis of the case and control groups showed that the wild-type GG can be regarded as the reference type, the rate distribution of $A G$ heterozygous mutants was higher in the case group than in the control group. Meanwhile, the distinction was significant. After logistic regression adjustment analysis, statistical difference was also noted. Stratification results revealed that the distribution rate of the AG type in the male population and in the non-drinking population was various in statistical data in these two groups. Thus, the AG type could be a danger factor. In the recessive model, the layout of AA homozygous mutants was not the same in statistical data. The danger of $\mathrm{GC}$ in the AA-type population was significantly lower than that in the AG+GG-type population. This result suggests that the rs2297441 polymorphism of the TNFRSF6B gene is also related to the danger of GC. Homozygous mutations could be a protective factor for $\mathrm{GC}$.

In sum, this case-control study demonstrated the significant association between the TNFRSF6B gene SNP and GC.
To the best of acknowledge, it could be treated as the first report on the risk of TNFRSF6B SNP associated with the danger of GC. This study also found a correlation between smoking and GC. For TNFRSF6B rs2297440, the TC genotype may be a risk factor for $\mathrm{GC}$ in people $<62$ years old. In the nondrinking population, the homozygous mutant of CC may be a protective factor for GC. In the drinking population, the TC type may be a risk factor, whereas the $\mathrm{TC}+\mathrm{CC}$ type dominated by $\mathrm{C}$ may be a protective factor. For TNFRSF6B rs2297441, the AG genotype may be a risk factor for $\mathrm{GC}$ in males and nondrinkers. The AA homozygous mutant may be a protective factor for GC.

This case-control study has several limitations. First, the patients and controls were enrolled from hospitals. Thus, inherent bias may have resulted in spurious findings. Second, the polymorphisms studied may not provide a comprehensive view of TNFRSF6B genetic variability. Fine mapping studies are required. Functional analyses to clarify the mechanisms behind TNFRSF6B associated disease susceptibility were lacking. In future studies, we will investigate the role of this SNP in the apoptosis and invasion capacity of GC cells. Third, the statistical power was limited because of the moderate sample size and absence of a validation cohort. In addition, gene-gene interaction and gene environment interactions play an important role in the pathogenesis of many diseases, especially chronic diseases. Helicobacter pylori (HP) infection is a common risk factor of GC. However, all patients with gastric cancer and health examiners in our hospital from 2013 to 2017 did not routinely test for HP because of hospital restrictions. As a result, we did not collect this information to analyze the association between TNFRSF6B SNP and HP. Other limitations included sampling of individuals of the same geographical region and race. Therefore, further studies considering different geographical locations and races and involving a large number of participants are necessary to confirm the results of the present study.

\section{Ethical Approval}

The research was approved by the Ethics Review Committee of Jiangsu University.

\section{Acknowledgments}

This work was supported by grants from Jiangsu Innovative Team Leading Talent Fund (CXTDC2016006, QNRC2016446), Jiangsu Provincial Key Research and Development Special Fund (BE2015666), Jiangsu 333 Talent Fund (BRA2020016), Jiangsu Six High Peak Talent Fund (WSW-205), Suqian Science and Technology Support 
Project Fund (S201721,K201907). In addition, the authors do appreciate all participants of this study sincerely.

\section{Disclosure}

The authors declare no conflicts of interest.

\section{References}

1. Smyth EC, Nilsson M, Grabsch HI, van Grieken NC, Lordick F. Gastric cancer. Lancet (London, England). 2020;396 (10251):635-648. doi:10.1016/S0140-6736(20)31288-5

2. Wang F, Shen L, Li J, et al. The Chinese Society of Clinical Oncology (CSCO): clinical guidelines for the diagnosis and treatment of gastric cancer. Cancer Commun. 2019;39(1):10. doi:10.1186/ s40880-019-0349-9

3. Song Z, Wu Y, Yang J, Yang D, Fang X. Progress in the treatment of advanced gastric cancer. Tumour Biol. 2017;39 (7):1010428317714626. doi:10.1177/1010428317714626

4. Tan H, Zhang S, Zhang J, et al. Long non-coding RNAs in gastric cancer: new emerging biological functions and therapeutic implications. Theranostics. 2020;10(19):8880-8902. doi:10.7150/ thno. 47548

5. Li-Sheng Chen S, Ching-Yuan Fann J, Sipeky C, et al. Risk prediction of prostate cancer with single nucleotide polymorphisms and prostate specific antigen. $J$ Urol. 2019;201(3):486-495. doi:10.1016/ j.juro.2018.10.015

6. Mueller AM, Pedré X, Killian S, David M, Steinbrecher A. The decoy receptor 3 (DcR3, TNFRSF6B) suppresses Th17 immune responses and is abundant in human cerebrospinal fluid. $J$ Neuroimmunol. 2009;209 (1-2):57-64. doi:10.1016/j.jneuroim.2009.01.024

7. Chen G, Rong M, Luo D. TNFRSF6B neutralization antibody inhibits proliferation and induces apoptosis in hepatocellular carcinoma cell. Pathol Res Pract. 2010;206(9):631-641. doi:10.1016/j. prp.2010.05.011

8. Yu W, Xu Y, Tao Y, et al. DcR3 regulates the growth and metastatic potential of SW480 colon cancer cells. Oncol Rep. 2013;30 (6):2741-2748. doi:10.3892/or.2013.2769

9. Zhao T, Xu Y, Ren S, et al. The siRNA silencing of DcR3 expression induces Fas ligand-mediated apoptosis in HepG2 cells. Exp Ther Med. 2018;15(5):4370-4378.

10. Ren Z, Zhu J, Gu H, et al. Decoy receptor 3 polymorphisms are not associated with the risk of esophageal cancer in a Chinese population. Biomarkers. 2014;19(4):340-344. doi:10.3109/1354750X.2014.915343

11. Zhang Y, Luo J, He R, et al. Expression and clinicopathological implication of DcR3 in lung cancer tissues: a tissue microarray study with 365 cases. Oncotargets Ther. 2016;9:4959-4968. doi:10.2147/OTT.S105225

12. Zhang Y, Huang S, Leng Y, et al. Effect of DcR3-specific siRNA on cell growth suppression and apoptosis induction in glioma cells via affecting ERK and AKT. Oncotargets Ther. 2016;9:5195-5202. doi:10.2147/OTT.S108395

Pharmacogenomics and Personalized Medicine

\section{Publish your work in this journal}

Pharmacogenomics and Personalized Medicine is an international, peer-reviewed, open access journal characterizing the influence of genotype on pharmacology leading to the development of personalized treatment programs and individualized drug selection for improved safety, efficacy and sustainability. This journal is indexed
13. Zhang X, Takata K, Cui W, et al. Protocadherin $\gamma$ A3 is expressed in follicular lymphoma irrespective of BCL2 status and is associated with tumor cell growth. Mol Med Rep. 2016;14(5):4622-4628. doi: $10.3892 / \mathrm{mmr} .2016 .5808$

14. Wei Y, Chen X, Yang J, et al. DcR3 promotes proliferation and invasion of pancreatic cancer via a DcR3/STAT1/IRF1 feedback loop. Am J Cancer Res. 2019;9(12):2618-2633.

15. Macher-Goeppinger S, Aulmann S, Wagener N, et al. Decoy receptor 3 is a prognostic factor in renal cell cancer. Neoplasia (New York, N Y). 2008;10(10):1049-1056. doi:10.1593/neo.08626

16. Bou-Dargham MJ, Liu Y, Sang QA, Zhang J, Seagroves T. Subgrouping breast cancer patients based on immune evasion mechanisms unravels a high involvement of transforming growth factor-beta and decoy receptor 3. PLoS One. 2018;13(12):e0207799. doi:10.1371/journal.pone.0207799

17. Ruan Y, Huang S, He D, et al. Effect of TNFRSF6B neutralization antibody on cell growth suppression and apoptosis induction in glioma cells. Neoplasma. 2015;62(4):574-581. doi:10.4149/ neo 2015069

18. Xiong G, Guo H, Wang K, et al. Polymorphisms of decoy receptor 3 are associated with risk of esophageal squamous cell carcinoma in Chinese Han. Tumour Biol. 2010;31(5):443-449. doi:10.1007/ s13277-010-0053-5

19. Dai L, Fu L, Liang Y, et al. A functional polymorphism rs2257440 in the gene DcR3 regulates its expression via MTF-1 in esophageal squamous cell carcinoma. Int $J$ Clin Exp Pathol. 2017;10 (11):11006-11013.

20. Fu Z, Chen S, Liu S, et al. DcR3 gene polymorphisms are associated with sporadic breast infiltrating ductal carcinoma in Northeast Chinese women. Oncotarget. 2016;7(36):57970-57977. doi:10.18632/oncotarget.11153

21. Shi G, Wu Y, Zhang J, Wu J. Death decoy receptor TR6/DcR3 inhibits $\mathrm{T}$ cell chemotaxis in vitro and in vivo. $J$ Immunol (Baltimore, Md: 1950). 2003;171(7):3407-3414. doi:10.4049/ jimmunol.171.7.3407

22. Chang Y, Chan Y, Jackson DG, Hsieh S. The glycosaminoglycan-binding domain of decoy receptor 3 is essential for induction of monocyte adhesion. $J$ Immunol (Baltimore, Md: 1950). 2006;176(1):173-180. doi:10.4049/jimmunol.176.1.173

23. Edfeldt K, Daskalakis K, Bäcklin C, et al. DcR3, TFF3, and midkine are novel serum biomarkers in small intestinal neuroendocrine tumors. Neuroendocrinology. 2017;105(2):170-181. doi:10.1159/ 000452891

24. Hu R, Liu W, Qiu X, et al. Expression of tumor necrosis factor- $\alpha$ induced protein 8 in stage III gastric cancer and the correlation with DcR3 and ERK1/2. Oncol Lett. 2016;11(3):1835-1840. doi:10.3892/ ol.2016.4133 on the American Chemical Society's Chemical Abstracts Service (CAS). The manuscript management system is completely online and includes a very quick and fair peer-review system, which is all easy to use. Visit http://www.dovepress.com/testimonials.php to read real quotes from published authors.

\title{
Dovepress
}

\author{
(1)
}

